\title{
Detection of composite damage by IR NDT using ultrasonic and optic excitation
}

\author{
Vladimír Dekýš ${ }^{1}$, Ondrej Štalmach ${ }^{1, *}$, Josef Soukup ${ }^{2}$, Alžbeta Sapietová ${ }^{1}$, and Lenka \\ Rychlíková ${ }^{2}$ \\ ${ }^{1}$ University of Zilina, Faculty of Mechanical Engineering, Department of Applied Mechanics, \\ Univerzitná 1, 01026 Zilina, Slovak Republic \\ ${ }^{2}$ University J. E. Purkyne in Usti nad Labem, Faculty of Mechanical Engineering, Institute of \\ Machinery and Energetics, Na Okraji 1001, 40096 Ústí nad Labem, Czech Republic
}

\begin{abstract}
The submitted paper presents experience with detection of damaged composites by means of an ultrasonic excitation system in comparison with an optic system of excitation. In case of the ultrasonic excitation system is a high-frequency signal modulated by lock-in frequency. For a selection of the carrier signal we measured a response of a sample to sweep excitation and a sample resonance was defined by means of STFT. Consequently, the method lock-in was applied for detection. In case of the optic excitation system we applied several suitably selected frequencies with an aim to obtain information from various depths of the measured object. Results of measurements are presented in composite samples of CFRP.
\end{abstract}

Keywords: Composite, infrared, thermography, NDT, lock-in, UTvis, OTvis

\section{Introduction}

Many applied processes of verification are based on detection of a response of a measured object to an outer environment. In the course of excitation, an object is supplemented by energy that is consequently transformed on the basis of the object properties and it is radiated into environment. If in the mentioned transformation do not origin detectable modifications in the object that would modify its properties and if we can detect and measure the radiated energy, then, simplistically, we may speak of a non-destructive testing. If we intend to detect inhomogeneities in an object structure, then it is necessary to select a suitable way of excitation and response detection.

In the paper we deal with detection of inhomogeneities of types of cracks, faults, gaps and holes in the composite object. These inhomogeneities may origin e.g., by a projectile impact on a composite plate, by an excessive compression of an object, by grinding a hole into an object or by any other artificial formation of a line defect $[1,2,3]$.

\footnotetext{
${ }^{*}$ Corresponding author: ondrej.stalmach@,fstroj.uniza.sk

Reviewers: Juraj Gerlici, Michat Śledziński
} 


\section{Applied equipment and method of processing of measured data}

In the paper we restrict a detection of radiated energy within the range of Mid-wave infrared (MWIR) spectrum by a cooled thermal detectable infrared camera FLIR SC7500 with a frame rate $383 \mathrm{~Hz}$ and a pixel resolution of $320 \times 256$.

Excitation is performed by an ultrasonic system UTvis and a system of optic excitation OTvis by the company edevis ${ }^{\circledR} \mathrm{GmbH}$.

UTvis enables to contact excitation of an object by means of sonotrodes in the frequency range of $15-25 \mathrm{kHz}$ while this signal is modulated by a low-frequency component $f_{\text {LOCKIN. }}$ The maximum system power is $2.2 \mathrm{~kW}$. A pressed sonotrode may induce local modifications in its environment. This causes a risk of damage of a tested object. If an inhomogeneity is analyzed, e.g., in a shape of a cavity, just below the surface, then a vibrant thin shell between the inhomogeneity and the surface of the tested object may damage. In case of a sensitive selection of excitation parameters we may avoid such destruction processes.

OTvis provides excitation of an object by means of halogen reflector lamps that periodically turn on to a required performance and turn off. Maximum power of reflector lamps is $2 \times 2.5 \mathrm{~kW}$. A period of the process is controlled by a frequency $f_{\mathrm{LOCKIN}}$. Band pass filters are used in the range of the wavelengths of MWIR. It is a contactless method that reduces a risk of mechanical damage of the tested object. However, a risk of damage increases in case of thermosensitive tested objects when long periods of excitation are applied and a heating of a sample is undesirable.

The sequence of frames was registered and processed by the lock-in method. This method is a specific form of Fourier Transform. In the case of the classical transformation the minimum frequency of the Fourier series is determined based on the number of samples and sampling frequency. For the lock-in method this minimum frequency is equal to the $f_{\text {LOCKIN }}$ frequency.

It is assumed that the system is stimulated by the load modulated by $f_{\text {LOCKIN }}$ and the response of the system $s(t)$ sufficiently is described by the harmonic function with the same

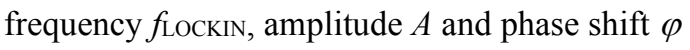

$$
\begin{gathered}
s(t)=A \sin \left(2 \pi f_{\text {LOCKIN }} t+\varphi\right)=a \cos \left(2 \pi f_{\text {LOCKIN }} t\right)+b \sin \left(2 \pi f_{\text {LOCKIN }} t\right), \\
a=A \sin (\varphi), b=A \cos (\varphi) .
\end{gathered}
$$

Let the amplitude of this response $s(t)$ is very small and the signal $S(t)$ being measured also contains a much higher noise $N(t)$, according to the relationship

$$
S(t)=s(t)+N(t)
$$

If $S(t)$ is expressed by Fourier's series than

$$
\begin{aligned}
S(\mathrm{t}) & =\frac{A_{0}}{2}+\sum_{k=1}^{+\infty} A_{k} \sin \left(2 \pi k f_{\text {LOCKIN }} t+\varphi_{k}\right)= \\
& =A_{1} \sin \left(2 \pi f_{\text {LOCKIN }} t+\varphi_{1}\right)+\left[\frac{A_{0}}{2}+\sum_{k=2}^{+\infty} A_{k} \sin \left(2 \pi k f_{l_{\text {LOCKIN }}} t+\varphi_{k}\right)\right]= \\
& =s(t)+N(t) .
\end{aligned}
$$

If $A=A_{l}$ and $\varphi=\varphi_{1}$, than the coefficients $a, b$ in (2) can be estimated by using the relationships of Discrete Fourier transformation

$$
a=\sum_{i=0}^{N-1} S_{i} \cos \left(2 \pi k f_{\text {LOCKIN }} \Delta t\right), \quad b=\sum_{i=0}^{N-1} S_{i} \sin \left(2 \pi k f_{\text {LOCKIN }} \Delta t\right)
$$


where $S_{i}$ is the $i$-th frame recorded by infrared camera (the frames are numbered $0, \ldots N-1$ ), $\Delta t$ is the period of frame frequency of camera. By using $a, b$, the amplitude $A$, phase $\varphi$, the real and the imaginary part of $s$ were determined for $f_{\text {LOCKIN. }}$.

The phase explains phase shift between reference excitation signal and response (periodic local change of temperature). The phase image is not disturbed by radiation reflected on the object, by variation of emissivity and it is insensitive to a locally uneven distribution of the applied thermal wave. The amplitude image can be disturbed by these factors, but amplitude and phase images can be useful. It depends on the situation. This technology allows detection of response radiation with a sensitivity of 100 to 1000 greater than the best thermal camera. In thermographic measurements, it is often very important to know or identify the emissivity of object, $[4,5,6]$. In general, determining this parameter may be complicated. This method avoids this problem.

In Fig. 1 there are systems UTvis (ultrasonic excitation, a sonotrode) and OTvis (optic excitation, lamps).
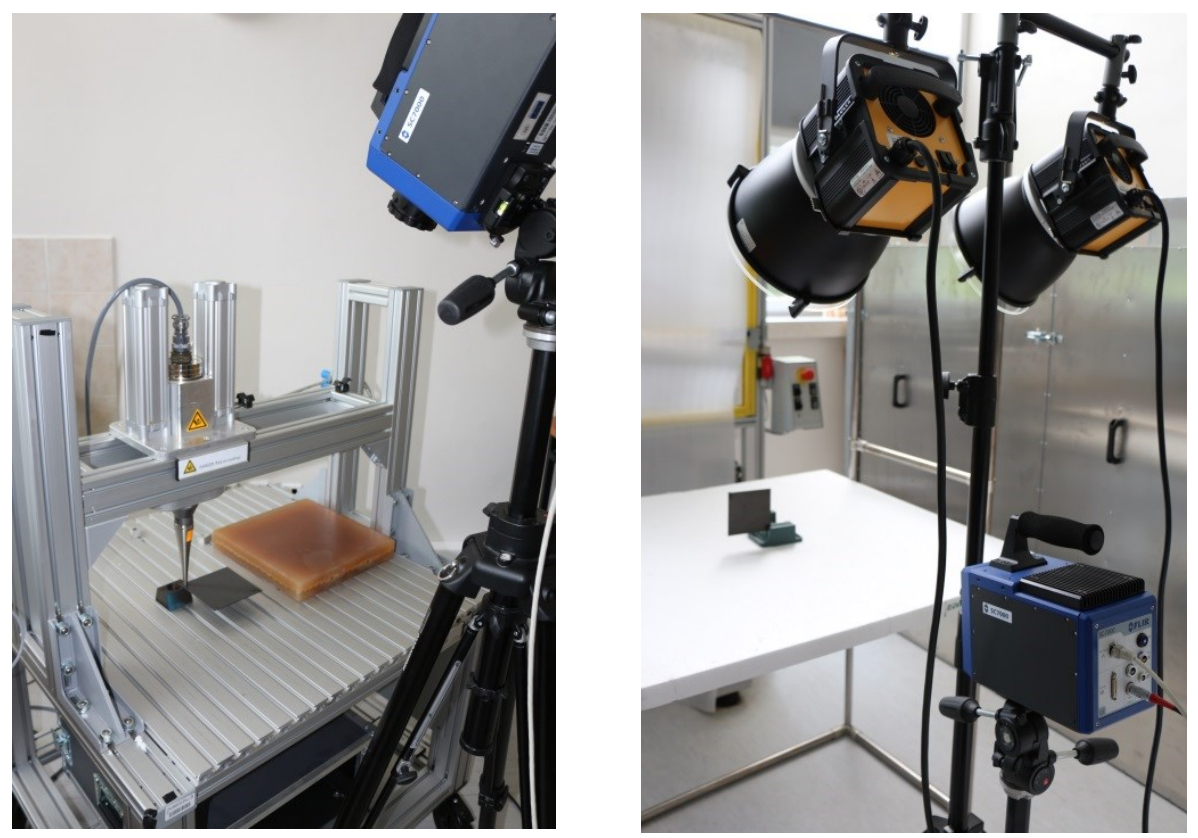

Fig. 1. Ultrasonic system UTvis (left) and optic system OTvis (right) together with infrared camera FLIR SC7500 applied in NDT

\section{Measured object}

An analysed object was a composite plate with carbon fibers placed horizontally on which a body falls in a vertical direction, from top to bottom. After its impact, the body rebounces and falls repeatedly. Several impacts (and consequent rebounds) were performed. In Fig. 2 there are photographs of the plate from the side of a fallen body (a front plate) and from the opposite side. 

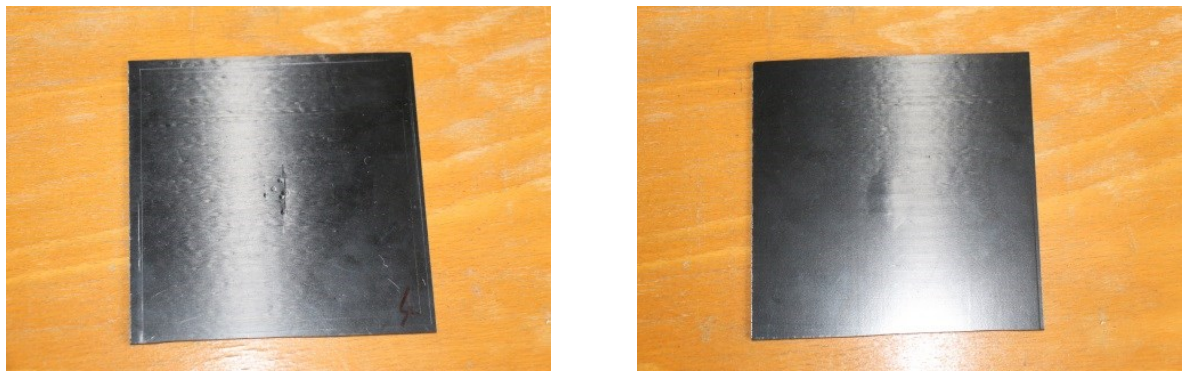

Fig. 2. A composite plate after an impact, left - from the side of the fallen body (front), right- from the opposite side (back)

In a suitable illumination (daylight) there is a visible crack in the front side of the plate with spots impressed by impacts. In the backside there is a visible bulge of the plate.

\section{Results of measurements}

As it was stated above, a measured system response $s(t)$ for excitation is a temperature equivalent to a radiation hitting a detector and before processing by means of lock-in it can be expressed by means of a reflection of temperature of the measured object Temperature. After processing by means of lock-in we obtain amplitudes $A$ and phases $\varphi$, according to (1), for every single pixel a of the detector of the camera. The outcome of the processing of measured data can be an amplitude image of a response of the measured object Amplitude and the phase image Phase. By presentation of a response by means of complex numbers we may express the processing outcome by means of a real image of the object response Real and imaginary one Imag, resp. combinations of the images Real/Imag or as a complex image Complex.

Results of measurements of the plate before impacts by means of UTvis are in Fig. 3, plate/front after impacts in Fig. 4 and plate/back after impacts in Fig. 5.
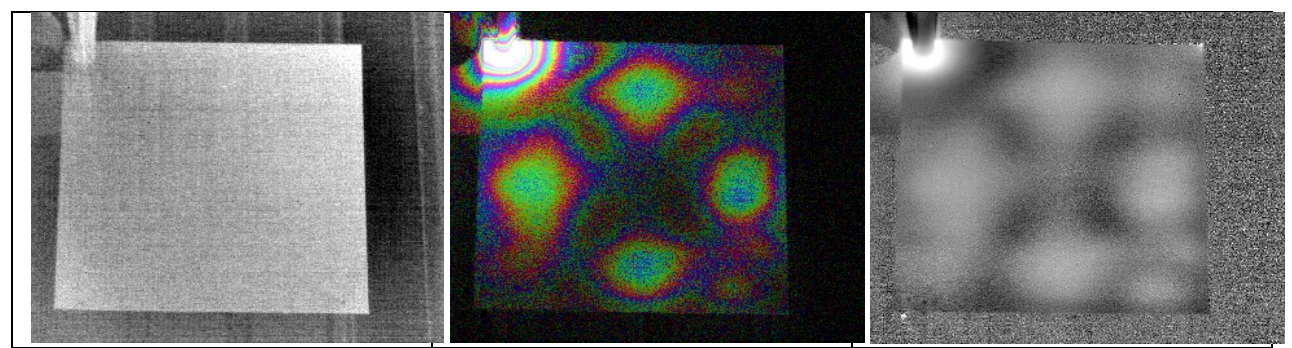

Fig. 3. UTvis. The composite plate before impacts, left - from the side of the fallen body (front), right - from the opposite side (back). Left - Temperature, in the centre - Complex, right - Phase 

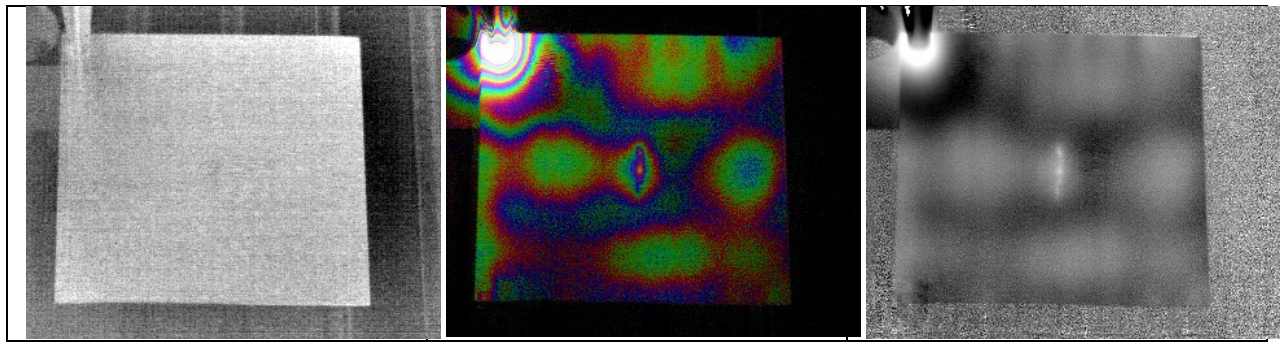

Fig. 4. UTvis. The composite plate after impacts, left - from the side of the fallen body (front), right from the opposite side (back). Left - Temperature, in the centre - Complex, right - Phase
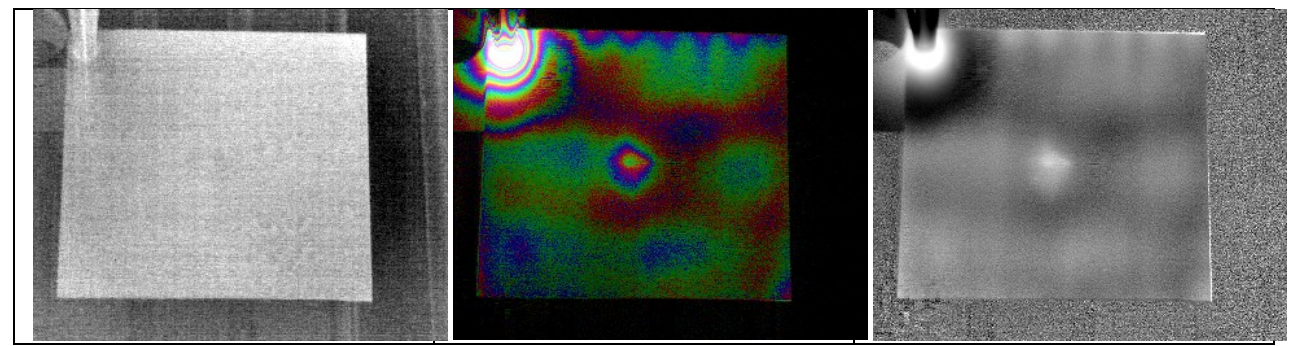

Fig. 5. UTvis. The composite plate after impacts, left - from the side of the fallen body (front), right from the opposite side (back). Left - Temperature, in the centre - Complex, right - Phase

In Figures 3, 4, 5 there is a dull image of inhomogeneity in Temperature. On the contrary, in images Complex and Phase is inhomogeneity sharp. It is necessary to suggest that in the image of Complex is suitable to increase original contrast and brightness by software means of image processing. (As it was in our case).

Similar measurements were performed also in case of optic excitation, by means of two $2.5 \mathrm{~kW}$ reflector lamps, the system OTVIS, Fig. 6. An image of inhomogeneity in Temperature is dull again, as a low distance signal/noise in measured very low thermal changes. In Complex it is similar as in this case a thermal wave from reflectors proceeds through the measured object and the object surface does not oscillate with elastic waves in a way as in the ultrasonic excitation, i.e., missing elastic waves do not activate corresponding oscillations. In Phase it is possible to detect inhomogeneity.

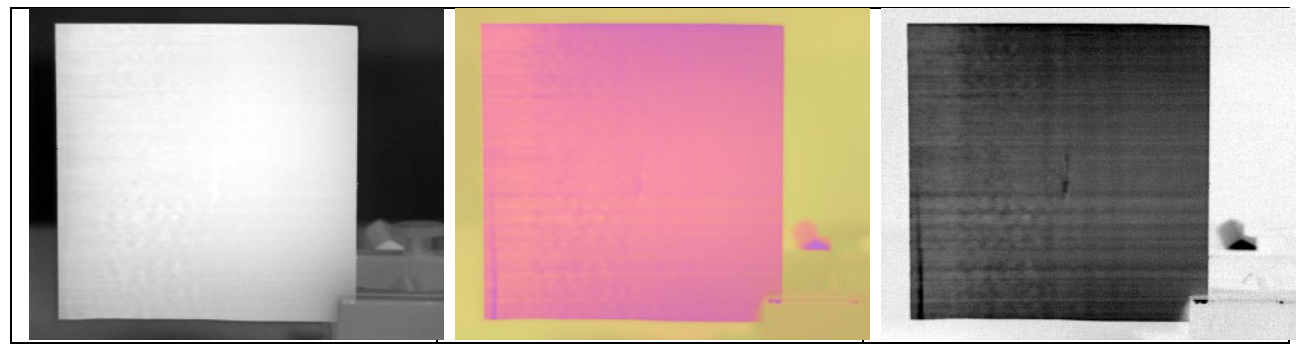

Fig. 6. OTvis. The composite plate after impacts, front. Left - Temperature, in the centre-Complex, right - Phase

In Fig. 7 and 8 there are images Phase for activating frequencies $3 \mathrm{~Hz}, 2 \mathrm{~Hz}, 1.23 \mathrm{~Hz}$ and $0.32 \mathrm{~Hz}$ for the plate (front) after impacts. With changing frequency of activating waves we obtain information of various depths of the measured object (with a lower frequency we get into a greater depth under the object surface, a thermal diffusive length increases). 

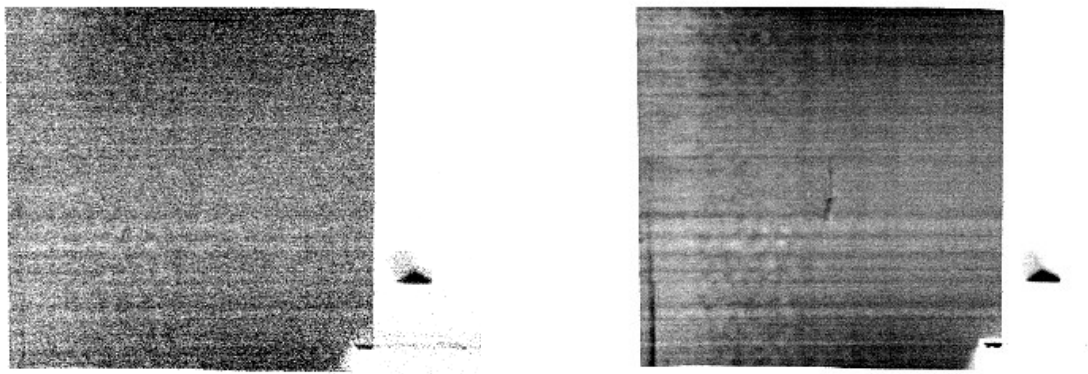

Fig. 7. OTvis. The composite plate after impacts, front. Left - activating frequency $3 \mathrm{~Hz}$, right activating frequency $2 \mathrm{~Hz}$
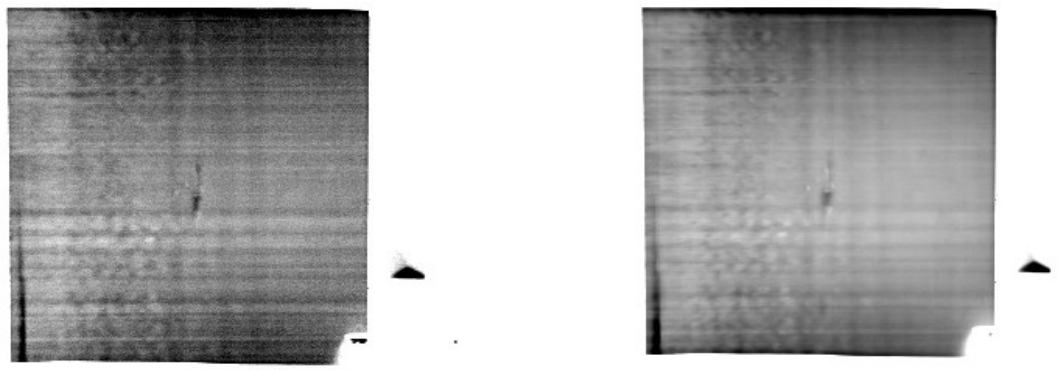

Fig. 8. OTvis. The composite plate after impacts, front. Left - activating frequency $1.23 \mathrm{~Hz}$, right activating frequency $0.32 \mathrm{~Hz}$

\section{Conclusion}

Generally, if optic excitation is regarded to be more efficient in an analysis of inhomogeneities in composites, then the paper presents a possibility of an ultrasonic excitation in the primary detection of inhomogeneity. We need to emphasize that this inhomogeneity was a crack that came to the surface of the analyzed composite.

The paper follows works in an area of composites at the Department of Applied Mechanics at Faculty of Mechanical Engineering of the University of Žilina [7, 8, 9, 10, 11].

This paper was supported by VEGA 1/0795/16, UJEP-IGS-2018-48-002-1 and UJEP-SGS-2018-48002-2 and by firm "TMV SS" Ltd. (Prague, Czech rep.) by borrowing the OTvis system.

\section{References}

1. M. Zimnoch, W. Oliferuk, M. Maj, Estimation of defect depth in steel plate using lockin ir thermography. Acta Mech. Autom. 4, 106-109 (2010)

2. R. Shrestha, K. Kisoo, K. Wontae, Investigation of Lock-in Infrared Thermography for Evaluation of Subsurface Defects Size and Depth. INT J PRECIS ENG MAN 16, 2255-2264 (2015)

3. Z. Vesely, M. Svantner, M., Application of IRNDT method for materials in wide range of thermal diffusivity. Quantitative Infrared Thermography, 895-901 (2016)

4. P. Honnerova, M. Honner, Survey of emissivity measurement by radiometric methods. Appl. Optics, Vol. 54, 669-683 (2015) 
5. P. Honnerova, J. Martan, M. Kucera, Martin, M. Honner, J. Hameury, New experimental device for high-temperature normal spectral emissivity measurements of coatings, MEAS SCI TECHNOL 25, Art.No. 095501 (2014)

6. M. Mauer, P. Kalenda, M. Honner, P. Vacikova, Composite fillers and their influence on emissivity. J Phys Chem Solids 73, 1550-1555 (2012)

7. M. Zmindak, Z. Pelagic, P. Pastorek, M. Mocilan, M. Vybostok. Finite element modelling of high velocity impact on plate structures. Procedia Eng. 136, 162-168 (2015)

8. M. Zmindak, Z. Pelagic, P. Pastorek, M. Bvoc, Analysis of High Velocity Impact on Composite Structures. Appl Mech Mater, Vol. 617, 104-109 (2014)

9. Z. Murcinkova, P. Novak, V. Kompis, M. Zmindak, Homogenization of the finite-length fibre composite materials by boundary meshless type method. ARCH APPL MECH 88, 789-804 (2018)

10. P. Pastorek, P. Novak, P. Kopas, M. Mocilan, Finite element analysis of bond behavior in a steel reinforced concrete structure strengthened carbon fibre reinforced polymer (CFRP) strips. METALURGIJA 56, 405-408 (2017)

11. L. Radziszewski, M. Saga, Modeling of non-elastic properties of polymeric foams used in sports helmets. Procedia Eng. 177, 314-317 (2017) 